\title{
Foreword
}

\section{Enhancing Clinical Outcomes in Hepatocellular Carcinoma}

\author{
Ghassan Abou-Alfa, MD ${ }^{1}$ \\ ${ }^{1}$ Department of Medicine, Memorial Sloan-Kettering Medical Center, \\ Weill Medical College at Cornell University, New York, New York
}

Semin Liver Dis 2013;33(S1):S1-S2.

Hepatocellular carcinoma (HCC) remains one of the most common solid-tumor malignancies and represents a global problem. Continued worldwide high incidence and rising incidence in the Western hemisphere is most likely related to chronic hepatitis $\mathrm{C}$, alcohol abuse, and diabetes- and obesity-associated nonalcoholic steatohepatitis (NASH). Understanding and managing "two diseases in one"-HCC and underlying liver dysfunction, which may promote hepatocarcinogenesis-is challenging and optimal management of HCC requires close collaboration of a multidisciplinary team consisting of several health care specialists. To diagnose HCC in an early, curable stage, careful surveillance of populations at risk is extremely important. Unfortunately, most HCC is diagnosed in the intermediate and advanced stages. With the last decade seeing expanded therapeutic options, patient outcomes continue to improve at a slow but steady pace. In recent years, there has been much research to better understand the complex, multistep pathogenesis of HCC and to identify potential new therapeutic targets and biomarkers in HCC.

The purpose of the three articles of this supplemental edition of the journal is to provide an update on the current state-of-the-art management of HCC, advances in the understanding of the molecular biology of HCC, and related emerging targeted therapies. The articles also feature future perspectives for further improvement of outcomes for patients with HCC.

In the first article, Jorge A. Marrero, MD, MS, from the University of Texas Southwestern in Dallas, reviews the epidemiology and risk factors for HCC. The importance of surveillance for patients at risk and diagnostic approaches when a liver nodule is detected are discussed. Prognosis and treatment decisions for a patient with HCC that are based on tumor stage, performance status, cancer-related symptoms, and liver function status are covered as well. Dr. Marrero also reviews all curable treatment options for patients with early
HCC, including resection, transplantation, and for selected patients, ablation. He also addresses locoregional treatment strategies for intermediate disease, such as embolization, chemoembolization, and radioembolization. Recognition that angiogenesis plays an important role in HCC development, growth, and progression, and evidence that the multitargeted kinase inhibitor sorafenib improved survival in advanced HCC represents a paradigm shift and begins the "targeted era" of HCC therapy.

In the second article, Richard S. Finn, MD, from the University of California in Los Angeles, provides an overview of research done to further elucidate the signaling pathways involved in HCC carcinogenesis. He also discusses trials of promising targeted agents for HCC that have reached later phases of clinical development. Additionally, signaling pathways other than angiogenesis that are involved in HCC pathogenesis are covered by Dr. Finn.

In addition to extensive research of potential new molecular targets for therapeutic intervention, the identification and validation of predictive biomarkers for appropriate treatment selection is essential. Recent advances in molecular genetics and new genomics tools are expected to facilitate identification of prognostic and predictive biomarkers in HCC. The third article in this supplement discusses future perspectives on areas for improvement and current efforts underway, particularly in terms of implementing global standards of care and identifying biomarkers to further personalize patient care.

Although the management of HCC is a challenge at every stage of disease, exciting advances in molecular biology and treatment have dramatically altered the clinical approach to this complex malignancy. Although several gaps remain in the appropriate management of HCC, ongoing efforts to personalize care at every stage of disease suggest a future of improved outcomes for patients with HCC.

\footnotetext{
Address for correspondence and reprint requests Ghassan AbouAlfa, MD, Department of Medicine, Memorial SloanKettering Medical Center, Weill Medical College at Cornell University, 300 East 66th Street, New York, NY 10065 (e-mail: Abou-alg@mskcc.org).
}

Issue Theme Enhancing Clinical Outcomes in Hepatocellular Carcinoma; Guest Editor, Ghassan Abou-Alfa, MD
Copyright @ 2013 by Thieme Medical Publishers, Inc., 333 Seventh Avenue, New York, NY 10001, USA. Tel: +1(212) 584-4662.
DOI http://dx.doi.org/ $10.1055 / \mathrm{s}-0033-1333630$. ISSN 0272-8087. 